\title{
Slow light and broadband coherent phonon generation
}

\author{
Zheng Wang*a,b Peter Rakich $^{\mathrm{c}}$, Charles Reinke ${ }^{\mathrm{c}}$, Ryan Camacho ${ }^{\mathrm{c}}$, Paul Davids ${ }^{\mathrm{c}}$ \\ ${ }^{a}$ Dept. of Electrical \& Computer Eng., The Univ. of Texas at Austin, Austin, TX USA 78712; \\ ${ }^{b}$ Research Lab. of Electronics, MIT, 77 Massachusetts Ave., Cambridge, MA USA 02139; \\ ${ }^{\mathrm{c} S}$ Sandia National Laboratories, PO Box 5800 Albuquerque, NM 87185-1082
}

\begin{abstract}
Recent advance in controlling optical forces using nanostructures suggests that nanoscale optical waveguides are capable of generating coherent acoustic phonons efficiently through a combination of radiation pressure and electrostriction. We discuss the critical roles of group velocity in such processes. This photon-phonon coupling would allow an acoustic intermediary to perform on-chip optical delay with a capacity $10^{5}$ greater than photonic delay lines of the same size.
\end{abstract}

Keywords: Slow light, stimulated Brillouin scattering, optical forces, radiation pressure, electrostriction, phonon-photon interaction

\section{INTRODUCTION}

Most nonlinear optical materials allow optical photons to be coupled to acoustic phonons at sufficiently high power via a third order Brillouin process. Phonons travel at the speed of sound, much slower than the speed of light, and can carry coherent information. Over the past several decades, electrostrictively mediated stimulated Brillouin processes have been well understood in bulk media and microscale waveguides, such as optical fibers. Such a process has allowed coherent phonon generation [1-3], novel slow-light cells [4,5], efficient and tailorable ultrahigh-frequency phonon generation [68], and signal processing schemes [9-11]. Cavity-based nano-optomechanical devices have recently been developed to enhance radiation pressures and the related Brillouin processes, in applications of phonon lasers[12], and phononic signal processing[13].

Here, we present a generalized multi-scale theory of photon-phonon coupling, valid at both micro- and nano-scales. This theory is used to rigorously treat traveling-wave SBS processes within the nanoscale waveguides: radically enhanced internal optical forces and high confinement phonon modes produced within such nanoscale systems are found to yield SBS gain coefficients which are $10^{5}-10^{6} \times$ larger than within typical silica optical fibers[14,15]. In contrast to microscale optical fibers and waveguides, both radiation pressure and electrostrictive optical forces play critical role in enhancing photon-phonon interactions at nanoscale, yielding a 100× stronger parametric gain than predicted by micro-scale SBS theories. Highly confined phonons, within nanoscale systems, are found to produce dominant forward-SBS processes, which are typically forbidden within guided-wave systems[16]. Comparison with exact relations reveals excellent agreement.

*zheng.wang@austin.utexas.edu; phone 1512 471-2991;

Advances in Slow and Fast Light V, edited by Selim M. Shahriar, Frank A. Narducci,

Proc. of SPIE Vol. 8273, 82731F · (c) 2012 SPIE · CCC code: 0277-786X/12/\$18 · doi: 10.1117/12.915285 


\section{RADIATION PRESSURE AND ITS SCALING IN NANOSCALE WAVEGUIDES}

In this section, we apply Response Theory of Optical Forces (RTOF), originally proposed in Ref. [17], to derive optical forces accurately from the amplitude and the phase responses: Forces produced by coherent electromagnetic fields internal to a mechanically-variable optical system are linked to the external optical responses of the system, especially to the change in group delay as a response to deformation of the waveguide. Unlike first-principle methods, such as surface integrals over the Maxwell stress tensor, RTOF method does not rely on the knowledge of the complex electromagnetic field profiles internal and often unique to the optical system. This reduction provides a unified understanding to a great many systems: provided that the optical responses are identical, these disparate electromagnetic systems produce exactly the same forces, even with drastically different internal field profiles. In the context of stepindex waveguides, we use its transmission function $S(q)=e^{i \phi(q)}$ to describe the optical response of a deformable waveguide. Here, $q$ is a generalized coordinate that represents the scaling parameter of the waveguide boundary $[18,19]$. The complex amplitude of the output waves, $b(q)$, is related to that of the incident waves $a$, as $b(q)=S(q) \cdot a$. The normalized wave amplitudes are related to the incident optical power $P^{i}$ and output power $P^{o}$ as $|a|^{2}=P^{i}$ and $|b|^{2}=P^{o} \cdot[20]$

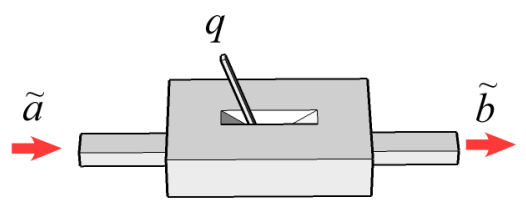

Fig. 1. Schematic of a single-port optical system with a single degree of freedom. q represents a geometric change to the optical waveguide.

For a single-port system illustrated in Figure 1, the optical response simplifies to a scalar form, where only phase response can be altered. Such a system include waveguides that are excited by a single spatial mode, which retains their translational symmetry when deform by optical forces. In comparison to the more general forms involving amplitude responses and scattering matrices[21], this simplification allows us to obtain the resulting optical force[17]

$$
F_{q}(\omega)=\frac{P^{i}}{\omega} \frac{\partial \phi(q, \omega)}{\partial q}
$$

Here we consider the simple case where only a single mechanical degree of freedom exists (Fig. 1), represented by a generalized coordinate q. Under such an optical force, one can consider a virtual displacement of the waveguide boundaries, such as a uniform scaling transformation of the form $\mathbf{r} \rightarrow \mathbf{r}^{\prime}=\mathbf{r} \cdot \beta$. An infinitesimal change in the scaling factor $\delta \beta$ is equivalent to a reduction in the critical dimensions of the waveguide, for example $a^{\prime}=a-\delta \beta \cdot a$. This infinitesimal geometric transformation corresponds to a change in total energy (or virtual work) $U_{E M}$, associated with the motion of the waveguide boundaries against optical and mechanical forces. The change in the electromagnetic energy is found to be

$$
\delta U_{E M}=-\int_{\delta w g} \mathbf{p} \cdot \mathbf{r} \delta \beta d S=-\frac{P_{i}}{\omega}\left(\frac{\partial \phi(q)}{\partial q}\right)_{\omega} \cdot \delta q
$$

Through the scaling law of Maxwell equation[15], one can further simplify this relation in terms of the group velocity and the phase velocity of the underlying mode. For a rectangular waveguide, seen in Figure 2, we can find the spatially averaged radiation pressure acting on the lateral and the horizontal boundaries to be

$$
\left(\bar{p}_{x}+\bar{p}_{y}\right)=\left(n_{g}-n_{p}\right)\left(P_{i} / c \cdot A_{w g}\right)
$$



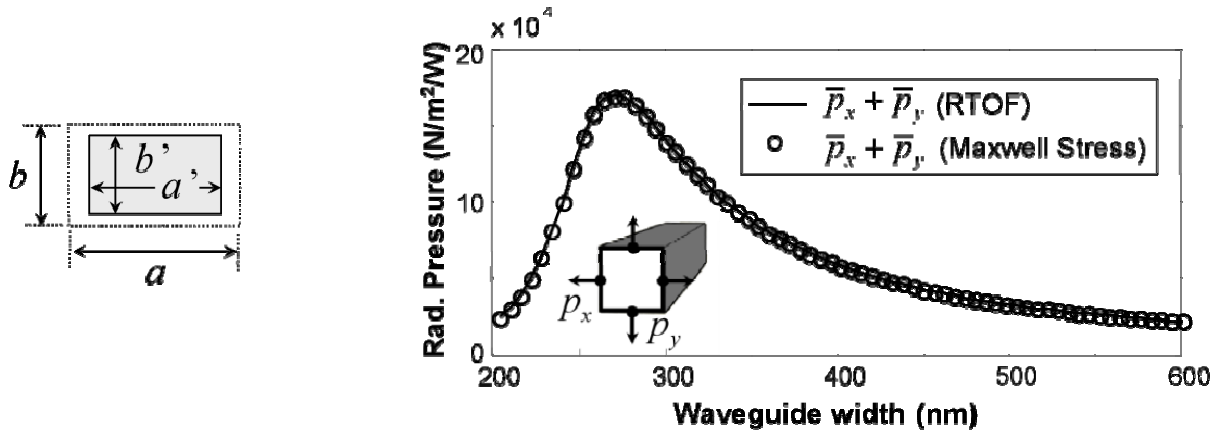

Fig. 2. Average radiation pressure on the boundary of a silicon rectangular waveguide as a function of waveguide width. The RTOF scaling law agrees well with numerical results from the Maxwell stress tensor.

For a silicon rectangular waveguide with a height of $315 \mathrm{~nm}$, we calculated the average pressure using the computed eigenvalues $\left(n_{p}, n_{g}\right)$ of the fundamental TE-like mode. The waveguide width is varied to show the effect of the geometric dispersion, where a maximum difference between $n_{p}$ and $n_{g}$ exists. The scaling relation derived from RTOF agrees perfectly with the Maxwell stress tensor calculation, and indicates that reducing group velocity in waveguide structures yield proportionally enhanced lateral pressures.

\section{PHONON GENERATION TRHOUGH COMBINED RADIATION PRESSURE AND ELECTROSTRICTION}

In this section, we analyze the rate of phonon generation through the combined effect of radiation pressure and electrostriction in a high index-contrast integrated photonic waveguide, with a lateral dimension on the order of hundreds of nanometers. In conventional microscale analyses of SBS, parametric coupling is computed from the nonlinear polarization currents associated with electrostrictively induced material density variations[22]. Reformulation of SBS theory is necessary at nanoscales, since the electrostrictive strain tensor[23], conventionally used to treat elastic-wave transduction, is no longer a valid construct for use in nano-optomechanical systems. Implicit in the development of the electrostrictive strain tensor are the bulk elastic material properties[23]; however, when confined to nanoscales, elastic waves no longer resemble bulk acoustic waves. Instead, one must formulate opto-mechanical coupling in terms of electrostrictive forces [14], and couple these forces to appropriate nano-mechanical elastic wave models. Furthermore, radiation pressures (which play a crucial role at nanoscales) must also be incorporated within models[14,15]. Conventional nonlinear polarization current treatments of SBS also become challenging to apply at nanoscales, as polarization currents must be computed where electric field and dielectric profiles are discontinuous.

To address these challenges, we develop a complete multi-scale SBS theory (MS-SBS) which treats photon-phonon coupling accurately at any length scale, provided that valid constitutive relations for dielectric (elastic) media exist. This theory avoids the difficulties of formulating nonlinear polarization currents within complex nano-opotmechanical systems by way of phononic domain calculations, and enables inclusion of all optically generated force within a single model.

The optical force densities generated by both radiation pressure and electrostriction are compared in Fig. 3, along with schematics which highlight the dominant forces acting on the waveguide. As described in Refs [14,15], the radiation pressure induced optical force density, $f_{j}^{r p}$, is computed from the divergence of the Maxwell stress-tensor. Such radiation-pressure-induced optical force densities are seen to be exactly localized to the discontinuous dielectric boundary of the step-index waveguide (vanishing within its volume). The force densities are seen in Fig. 3 a-d (units of $N / m^{3} / W$ ), coinciding with a vanishing longitudinal force density, $f_{z}^{r p}$. Such forces correspond to average $\mathrm{x}$ - and $\mathrm{y}-$ directed radiation pressures (stresses) of $1.2 \times 10^{5} \mathrm{~N} / \mathrm{m}^{2} / \mathrm{W}$ and $4.4 \times 10^{4} \mathrm{~N} / \mathrm{m}^{2} / \mathrm{W}$ respectively. Due to the large fractional energy density carried by longitudinal $\left(\mathrm{E}_{\mathrm{z}}\right)$ electric field and the high waveguide dispersion, radiation 
pressures can be seen to be $10^{2}-10^{4} \times$ larger than within optical fibers[15], yielding forces and stresses exceeding those of electrostriction[14].

The field induced electrostrictive stress distribution produced by the TE-like mode can be rigorously formulated in terms of the photoelastic tensor components of the core medium using the principle of virtual work[14]. Within cubic media such as silicon, the electrostrictively induced body stress becomes $\sigma_{k l}^{e s}=-\frac{1}{2} \grave{\mathrm{o}}_{o} n_{1}^{4} p_{i j k l} \cdot E_{i} E_{j}$ [14], yielding spatially averaged $\sigma_{x x}^{e s}$ and $\sigma_{y y}^{e s}$ stress components of $-3.3 \times 10^{4} \mathrm{~N} / \mathrm{m}^{2} / \mathrm{W}$ and $2.1 \times 10^{4} \mathrm{~N} / \mathrm{m}^{2} / \mathrm{W}$ respectively; such stress values are comparable to those produced by radiation pressure. The electrostrictively induced force density is computed from the divergence of $\sigma_{k l}^{e s}$ as $f_{l}^{e s}=-\partial_{k} \sigma_{k l}^{e s}$, yielding the computed transverse force distributions seen in Fig. 3 e-h, and a vanishing longitudinal force density, $f_{z}^{e s}$. In contrast to radiation pressure, electrostriction produces large optical forces within the waveguide volume and at its boundaries.
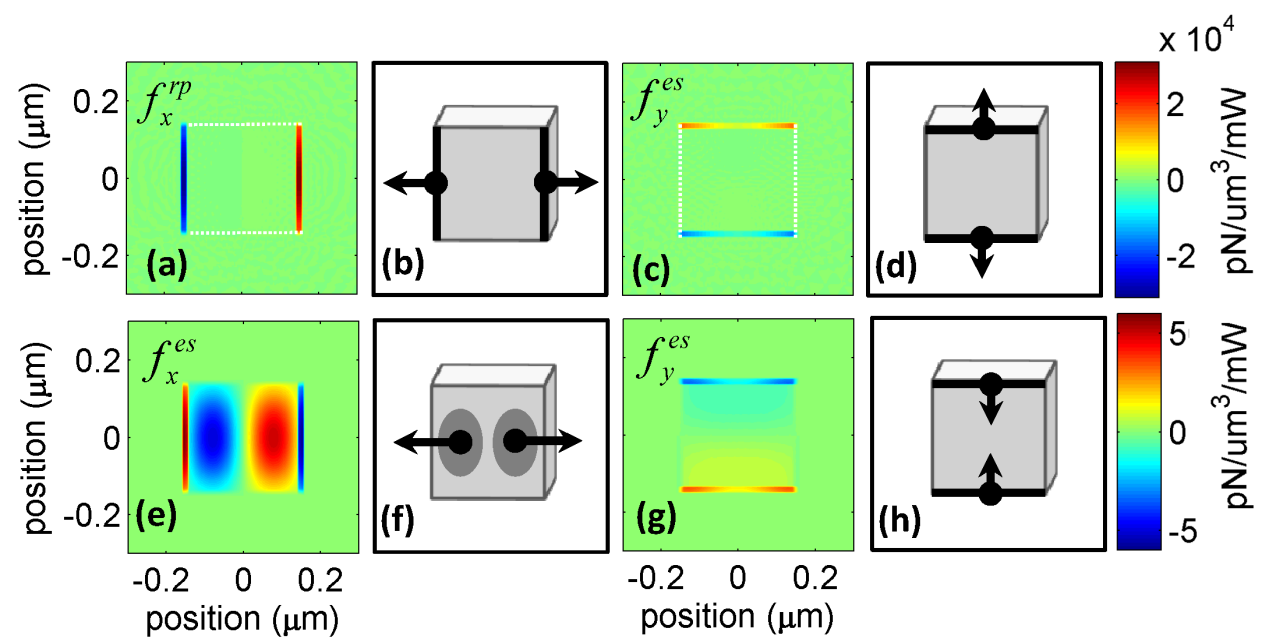

Fig. 3. (a) and (c) show time-averaged force densities $\mathrm{f}_{\mathrm{x}}^{\mathrm{rp}}$ and $\mathrm{f}_{y}^{\mathrm{rp}}$ generated by radiation pressure. (b) and (d) are schematics showing dominant forces in plots (a) and (c). (e) and (g) show time-averaged force densities $\mathrm{f}_{\mathrm{x}}^{\text {es }}$ and $\mathrm{f}_{\mathrm{y}}^{\text {es }}$ generated by electrostriction. (f) and (h) are schematics showing dominant forces in plots (e) and (g).

Through Brillouin processes, a high frequency pump photon of frequency, $\omega_{\mathrm{p}}$, produces a red-shifted Stokes photon $\left(\omega_{\mathrm{s}}\right)$ and an acoustic phonon $(\Omega)$ via a third order parametric process. Energy and momentum conservation require that $\omega_{p}=\omega_{s}+\Omega$, and $\mathbf{k}_{\mathbf{p}}=\mathbf{k}_{\mathbf{s}}+\mathbf{K}$, where $\mathbf{k}_{\mathbf{p}}, \mathbf{k}_{\mathbf{s}}$ and $\mathbf{K}$ are the pump, Stokes and phonon wave-vectors respectively. For waveguides, translational invariance limits the pump and Stokes photon (and phonon) momenta to a single axis. Thus, the only possible scattering processes are forward SBS (FSBS), with co-linear Stokes and pump waves, and backward SBS (BSBS) where Stokes and pump waves are contra-directionally coupled. Except in a few special cases[24,25], forward SBS is generally impossible to observe. However, within nano-scale waveguides, we show that FSBS processes are no longer forbidden.

The magnitude of photon-phonon coupling through Brillouin processes can be completely modeled by way of rigorously coupled electromagnetic and elastic-wave models. Through such models, optical forces mediate the coupling of energy between optical and phononic domains. Growth of the parametrically generated optical Stokes wave is described by the canonical relation[22],

$$
d P_{s} / d z=G_{B} \cdot P_{p} P_{s}
$$


Here, $P_{s}\left(P_{p}\right)$ is the power carried by the guided Stokes-wave (or pump-wave), and $G_{B}$ is the stimulated Brillouin scattering gain coefficient.

We assume that optical powers (particle fluxes) $P_{p}\left(\Phi_{p}\right), P_{s}\left(\Phi_{s}\right)$, corresponding to optical pump $\left(\omega_{p}\right)$ and a Stokes waves $\left(\omega_{s}\right)$ are coupled by acoustic phonons of frequency $(\Omega)$ and power (particle flux) $\mathrm{P}_{\Omega}\left(\Phi_{\Omega}\right)$. In describing the parametric conversion, we invoke particle conservation (the Manley-Rowe relations) yielding,

$$
d \Phi_{s} / d z=d \Phi_{\Omega} / d z=-d \Phi_{p} / d z
$$

This equation is applicable to non-dissipative systems where both photon and phonon fluxes are conserved.

If phonon dissipation is present, particle flux is no longer conserved. However, particle conservation can be used to determine phonon generation rates, despite high phonon losses. For instance, the rate of Stokes photon generation $\left(\delta \mathrm{R}_{\mathrm{s}}\right)$ within a short segment of waveguide, $\delta z$, is $\delta R_{s}=\left(d \Phi_{s} / d z\right) \cdot \delta z$. In the limit of small , Eq. (4) becomes

$$
d P_{s} / d z=\left[\delta R_{\Omega} / \delta z\right] \cdot\left(\hbar \omega_{s}\right)=G_{B} \cdot P_{p} \cdot P_{s} .
$$

Here, $P_{s}=\Phi_{s} \hbar \omega_{s}$ was used, where $\hbar$ is Planck's constant. From the time-varying optical force density $\left(\mathbf{f}_{\mathbf{\Omega}}(\mathbf{r}, t)\right)$, produced by the interference between pump and stokes waves, $\delta R_{\Omega}$ is computed from the generated elastic wave power as

$$
\delta R_{\Omega}=\frac{1}{\hbar \Omega} \cdot \int_{\delta V}<\mathbf{f}_{\Omega}(\mathbf{r}, t) \cdot \dot{\mathbf{u}}(\mathbf{r}, t)>d V
$$

Here, $\dot{\mathbf{u}}(\mathbf{r}, t)$ is the displacement velocity distribution of the elastic body in response to $\mathbf{f}_{\mathbf{\Omega}}(\mathbf{r}, t),<\ldots>$ denotes time the average over a period of harmonic motion, and integration is taken over, $\delta V$, the volume of the waveguide segment. Hence, in the limit of small $\delta z, \mathrm{G}_{B}$ is given by

$$
G_{B}(\Omega)=\frac{1}{\delta z} \frac{\omega_{s}}{\Omega} \cdot \frac{1}{P_{p} \cdot P_{s}} \int_{\delta V}<\mathbf{f}_{\mathbf{\Omega}}(\mathbf{r}, t) \cdot \dot{\mathbf{u}}(\mathbf{r}, t)>d V
$$

This remarkably simple expression treats photon-phonon coupling at any length scales, provided that valid constitutive relations for dielectric and elastic media exist. This result is applicable to both translationally invariant and periodic waveguides of arbitrary form. Note, $\mathbf{f}_{\mathbf{\Omega}}(\mathbf{r}, t)$ is a generalized optical force density, including all or any optical forces forces (e.g. radiation pressure, electrostriction, magnetostriction), as discussed in the earlier sections.

Limiting our attention to the special case of a translationally invariant waveguide, it is convenient to express the force (velocity) distribution in complex notation as

$$
\begin{aligned}
& \mathbf{f}_{\mathbf{\Omega}}(\mathbf{r}, t)=\operatorname{Re}\left[\tilde{\mathbf{f}}_{\mathbf{\Omega}}(\mathbf{x}, \mathbf{y}) \cdot e^{i(K z-\Omega t)}\right] \\
& \dot{\mathbf{u}}_{\mathbf{\Omega}}(\mathbf{r}, t)=\operatorname{Re}\left[\dot{\tilde{\mathbf{u}}}_{\mathbf{\Omega}}(\mathbf{x}, \mathbf{y}) \cdot e^{i(K z-\Omega t)}\right]
\end{aligned}
$$

Defining a power normalized force density, $\tilde{\mathbf{f}}_{\mathbf{K}}^{\mathbf{n}}(\mathbf{x}, \mathbf{y})$, where $\tilde{\mathbf{f}}_{\mathbf{\Omega}}(\mathbf{x}, \mathbf{y})=\tilde{\mathbf{f}}_{\mathbf{K}}^{\mathbf{n}}(\mathbf{x}, \mathbf{y}) \cdot 2 \cdot P_{s} P_{p}$, Eq. (7) simplifies to 


$$
G_{B}(\Omega)=\frac{\omega_{s}}{\Omega} \cdot \int_{w g} \operatorname{Re}\left[\tilde{\mathbf{f}}_{\mathbf{K}}^{\mathbf{n}}(\mathbf{x}, \mathbf{y}) \cdot \dot{\tilde{\mathbf{u}}}^{*}(\mathbf{x}, \mathbf{y})\right] \cdot d A
$$

Eq. (8) is a surprisingly simple and general exact solution for SBS within translationally invariant waveguides of arbitrary cross-section. Next, we consider the optical forces within nanoscale waveguides similar to Fig. 3.

We have previously shown that both radiation pressure and electrostrictive forces grow to tremendous values within high refractive index waveguides at nanoscales[14,15]. Due to the large fractional energy density carried by the longitudinal $E_{z}$ electric field, and the high waveguide dispersion, radiation pressures are $10^{2}-10^{4} \times$ larger than within optical fibers, yielding forces and stresses exceeding those of electrostriction.

The distributions of electrostrictive and radiation pressure induced forces, within nanoscale waveguides, are distinct. Radiation pressure induced optical forces $\left(\mathbf{f}^{\mathbf{r p}}\right)$ are exactly localized to the discontinuous dielectric boundary of the step-index waveguide[14,15], while the electrostrictive forces $\left(\mathbf{f}^{\mathbf{r p}}\right)$ are determined by the tensor photoelastic material properties, producing complex spatial distributions within the waveguide volume. Both depend sharply on nanoscale waveguide geometry and yield vanishing longitudinal forces under single mode excitation of a translationally invariant waveguide[14,15].

Next we consider silicon waveguide geometry (mode-field) seen in Fig. 3, corresponding to a core (cladding) refractive index of $\mathrm{n}_{1}=3.5\left(\mathrm{n}_{2}=1\right)$ and material photoelastic constants $\left[p_{11}, p_{12}, p_{44}\right]=[+0.09,-0.017,-0.054]$ consistent with those of bulk silicon. Here, $p_{i j}$ are the photoelastic tensor components $\left(p_{i j k l}\right)$ represented in contracted notation, and the [100] crystal symmetry direction (of this cubic system) coincides with the $\mathbf{x}$-axis.

In treating the SBS within this waveguide, the complex time-harmonic optical force distributions, $\tilde{\mathbf{f}}_{\mathbf{\Omega}}(\mathbf{x}, \mathbf{y})=\tilde{\mathbf{f}}_{\mathbf{K}}^{\mathbf{n}}(\mathbf{x}, \mathbf{y}) \cdot 2 \cdot P_{s} P_{p}$, were computed from electromagnetic FEM models (as in Ref [14,15]), and used to drive an elastic wave FEM model to compute $G_{B}(\Omega)$ versus frequency of excitation. For simplicity, a constant phononic material loss-Q of 1000 is assumed. Since both the electrostriction and radiation pressure produce symmetric force distributions, only symmetric elastic waves can be excited through either FSBS or BSBS processes. The first five symmetric elastic modes are plotted in Fig. 4b, for comparison FSBS and BSBS gain spectra of Fig. 4a and c.

Through FSBS processes $\mid \mathbf{K} \cong \Omega / v_{g}$, where $v_{g}$ is the group velocity of the optical mode within the waveguide. Since $|\mathbf{K}|$ is nearly zero, transverse optical forces from radiation pressure and electrostriction dictate the parametric photonphonon coupling. In stark contrast to microscale systems, this suspended waveguide produces high confinement guided elastic waves at $\mid \mathbf{K}=0$, with vanishing group velocity. Through FSBS, strong coupling to the S2 and S5 elastic modes are found (see colored insets of Fig. 4a).

In constrast, for BSBS processess, phonon wave-vector $|\mathbf{K}| \cong 2 \cdot\left|\mathbf{k}_{\mathbf{p}}\right|$, results in longitudinal electrostrictive forces of comparable magnitude to the transverse forces generated by both radiation pressure and electrostriction. In this case, simulations yield the SBS gain spectrum seen in Fig. 4c. Again, the separate contributions to the SBS gain produced by electrostriction (blue) and radiation pressure (green) are shown to constructively interfere at $13.8 \mathrm{GHz}$ to yield a radically enhanced SBS gain of $2.4 \times 10^{4} \mathrm{~m}^{-1} \mathrm{~W}^{-1}$. The coherent combination of these forces produce efficient coupling to the S1 and S4 modes of Fig. 4b. 

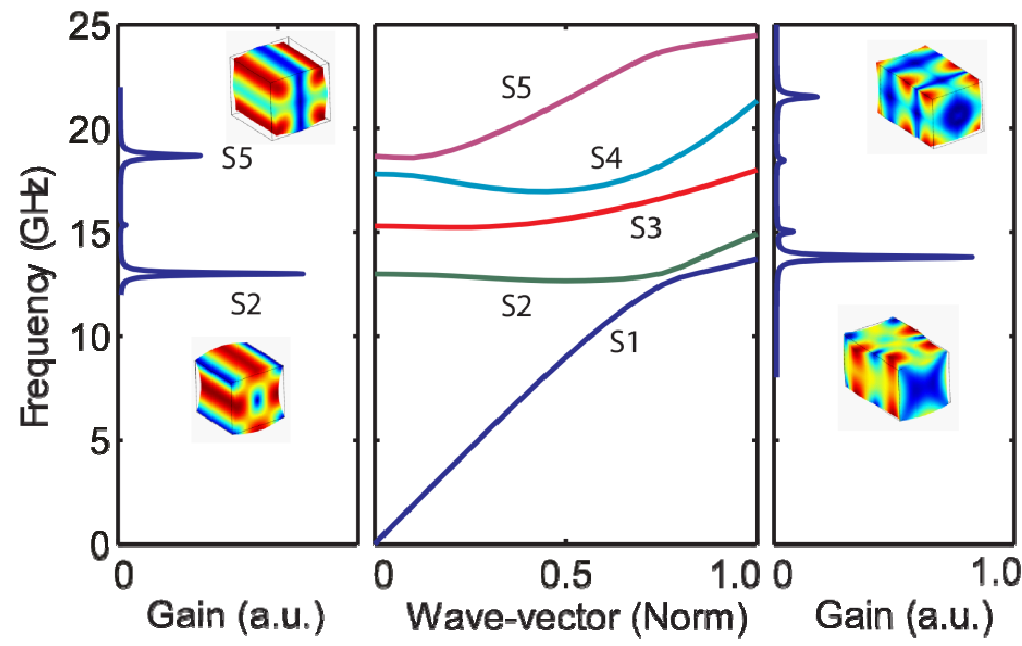

Fig. 4. Computed SBS gain (a.u.) for FSBS (a) and BSBS (c) processes. Both gain spectra are dictated by the elastic wave dispersion (b). Panel (b) shows the guided elastic wave dispersion for symmetric modes (labeled S1-S5). Here the wavevector is normalized to . Colored insets: displacement amplitudes and shape deformation of the dominant S1, S2, S4 and S5 elastic modes excited.

Figure 5 compares the peak BSBS gain computed via this milti-scale SBS theory with microscale SBS theories [16] for waveguide dimensions between $0.3-10 \mathrm{~m}$. Perfect agreement is seen for dimensions greater than $1 \mathrm{~m}$; however, for smaller dimensions, microscale SBS theories break down as both elastic modes and optical forces change radically at nanoscales, causing deviation from the predictions of approximate bulk acoustic wave models. As evidence, the peak gain resulting from coupling to transverse elastic waves (blue), which are of negligible impact at microscales, yielding the dominant BSBS interaction below 0.8 microns. This deviation results from the large transverse forces of radiation pressure and electrostricion, revealing that SBS gain cannot be estimated based on intrinsic properties of the waveguide material (photoelastic constants) at nanoscales.

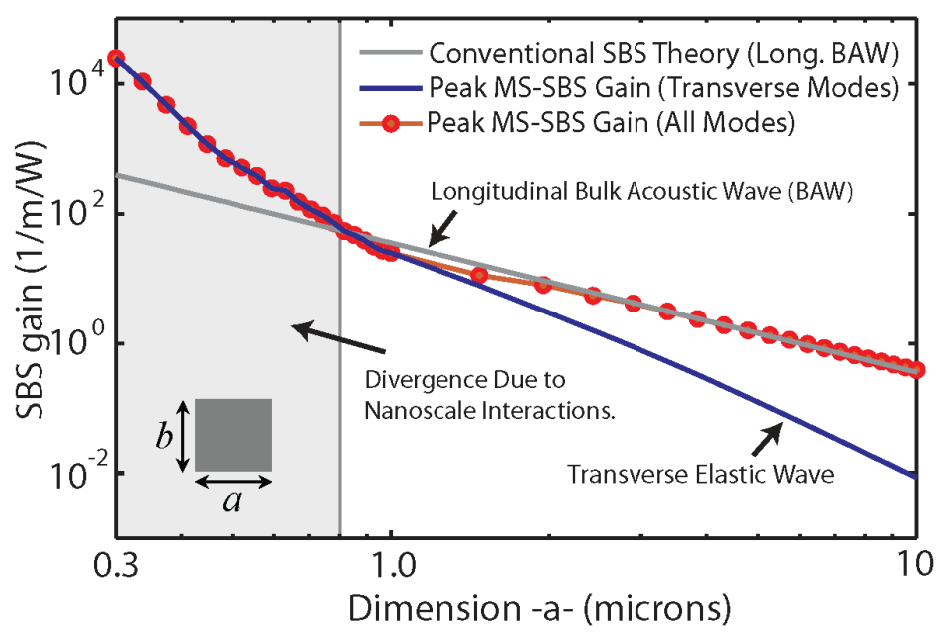

Fig. 5. Computed backward SBS gain versus waveguide dimension $a$, for

. Computed SBS gain (a.u.) for FSBS (a) and BSBS (c) processes. Both gain spectra are dictated by the elastic wave dispersion (b). Panel (b) shows the guided elastic wave dispersion for symmetric modes (labeled S1-S5). Here the wavevector is normalized to Colored insets: displacement amplitudes and shape deformation of the dominant S1, S2, S4 and S5 elastic modes excited. 
Note, the velocity amplitude of the elastic displacement field, and therefore $\mathrm{G}_{\mathrm{B}}$, increase linearly with phononic loss- $\mathrm{Q}$ (quadratically on the optical force). Furthermore, the thermoelastic damping limit of bulk silicon corresponds to $Q \cong 4,000(Q \cong 40,000)$ at $13 \mathrm{GHz}$ frequencies about $300 \mathrm{~K}(4 \mathrm{~K})$, indicating that $\mathrm{G}_{\mathrm{B}}$ could reach values of $9.6 \times 10^{4} \mathrm{~m}^{-1} \mathrm{~W}^{-1}\left(9.6 \times 10^{5} \mathrm{~m}^{-1} \mathrm{~W}^{-1}\right)$ under ideal experimental conditions. Such radically enhanced SBS processes are $10^{5}-10^{6} \times$ larger than within typical silica optical fibers, meaning that a nanoscale waveguide of $100 \mu \mathrm{m}$ length could produce SBS nonlinearities comparable to 10-100 meters of silica fiber.

\section{INTRODUCTION}

In conclusion, we have developed a generalized multi-scale theory of photon-phonon coupling (or stimulated Brillouin processes), applicable at any length scale, provided that valid constitutive relations for dielectric and elastic media exist. We applied this theory to traveling-wave SBS processes within the nanoscale waveguides, revealing that radically enhanced internal optical forces and high confinement phonon modes produced within nanoscale systems yield SBS gain coefficients which are $10^{5}-10^{6} \times$ larger than within typical silica optical fibers and $100 \times$ stronger parametric gain than predicted by micro-scale SBS theories, paving the way for on-chip coherent phonon generation with a bandwidth relevant to signal processing and optical buffering. Localized phononic resonances, within nanoscale systems, were shown to produce dominant forward-SBS processes, typically forbidden within guided-wave systems.

\section{REFERENCES}

[1] E. P. Ippen, "Stimulated Brillouin scattering in optical fibers," Applied Physics Letters 21, 539 (1972).

[2] R. Chiao, C. Townes, and B. Stoicheff, "Stimulated Brillouin Scattering and Coherent Generation of Intense Hypersonic Waves," Physical Review Letters 12, 592-595 (1964).

[3] T. J. Kippenberg and K. J. Vahala, "Cavity optomechanics: back-action at the mesoscale," Science 321, 1172-1176 (2008).

[4] Y. Okawachi, M. Bigelow, J. Sharping, D. Gauthier, R. Boyd, Z. Zhu, A. Schweinsberg, and A. Gaeta, “Tunable All-Optical Delays via Brillouin Slow Light in an Optical Fiber," Physical Review Letters 94, 1-4 (2005).

[5] M. González Herráez, K. Y. Song, and L. Thévenaz, "Arbitrary-bandwidth Brillouin slow light in optical fibers," Optics Express 14, 1395 (2006).

[6] P. Dainese, P. S. J. Russell, N. Joly, J. C. Knight, G. S. Wiederhecker, H. L. Fragnito, V. Laude, and a. Khelif, "Stimulated Brillouin scattering from multi-GHz-guided acoustic phonons in nanostructured photonic crystal fibres," Nature Physics 2, 388-392 (2006).

[7] K. A. Nelson, "Stimulated Brillouin scattering and optical excitation of coherent shear waves," Journal of Applied Physics 53, 6060 (1982).

[8] R. M. Shelby, M. D. Levenson, and P. W. Bayer, "Guided acoustic-wave Brillouin scattering," Physical Review B 31, 5244-5252 (1985).

[9] M. Tomes and T. Carmon, "Photonic Micro-Electromechanical Systems Vibrating at X-band (11-GHz) Rates," Physical Review Letters 102, 20-23 (2009).

[10] M. L. Povinelli, M. Loncar, M. Ibanescu, E. J. Smythe, S. G. Johnson, F. Capasso, and J. D. Joannopoulos, "Evanescent-wave bonding between optical waveguides," Optics Letters 30, 3042 (2005).

[11] A. Mizrahi and L. Schächter, "Two-slab all-optical spring," Optics Letters 32, 692 (2007).

[12] I. S. Grudinin, H. Lee, O. Painter, and K. J. Vahala, "Phonon Laser Action in a Tunable Two-Level System," Nature 083901, 2-5 (2010).

[13] P. T. Rakich, M. A. Popović, M. Soljačić, and E. P. Ippen, "Trapping, corralling and spectral bonding of optical resonances through optically induced potentials," Nature Photonics 1, 658-665 (2007).

[14] P. T. Rakich, P. Davids, and Z. Wang, "Tailoring optical forces in waveguides through radiation pressure and electrostrictive forces.," Optics express 18, 14439-14453 (2010).

[15] P. T. Rakich, Z. Wang, and P. Davids, "Scaling of optical forces in dielectric waveguides: rigorous connection between radiation pressure and dispersion.," Optics letters 36, 217-219 (2011).

[16] G. Agrawal, Nonlinear Fiber Optics, 4th ed., p. 552, Academic Press (2006).

[17] P. T. Rakich, M. Popović, and Z. Wang, "General treatment of optical forces and potentials in mechanically variable photonic systems.," Optics express 17, 18116-18135 (2009). 
[18] H. A. Haus, Waves and fields in optoelectronics, p. xii, 402, Prentice-Hall, Englewood Cliffs, NJ (1984).

[19] D. M. Pozar, Microwave Engineering, 2nd ed., John Wiley, New York (1998).

[20] H. A. Haus, "Mirrors and Interferometers," in Waves and fields in optoelectronics, pp. 55-80, Prentice-Hall, Englewood Cliffs, NJ (1984).

[21] Z. Wang and P. Rakich, "Response theory of optical forces in two-port photonics systems : a simplified framework for examining conservative and non-conservative forces," Optics Express 19, 22322-22336 (2011).

[22] R. Boyd, Nonlinear optics, Academic Press, Boston (1992).

[23] A. Feldman, "Relations between electrostriction and the stress-optical effect," Physical Review B 11, 5112-5114, APS (1975).

[24] A. Corvo and A. Gavrielides, "Forward stimulated Brillouin scattering," Journal of Applied Physics 63, 5220 (1988).

[25] A. Matsko, Y. Rostovtsev, M. Fleischhauer, and M. Scully, "Anomalous Stimulated Brillouin Scattering via Ultraslow Light,” Physical Review Letters 86, 2006-2009 (2001). 